\title{
CARACTERIZACIÓN PETROGRÁFICA, OUIIMICA Y EDAD Ar-Ar DE CUERPOS PORFÍDICOS INTRUSIVOS EN LA FORMACIÓN SALDAÑA
}

\author{
PETROGRAPHICAL AND CHEMICAL CHARACTERIZATION AND \\ Ar-Ar AGE DETERMINATION OF INTRUSIVE PORPHYRITIC BODIES \\ IN THE SALDAÑA FORMATION
}

\author{
Gabriel Rodríguez García ${ }^{1}$ \\ *Email de correspondencia: grodriguez@sgc.gov.co
}

Recibido: 8/08/2017

Aceptado: 31/10/2017

Publicado en línea: 20/04/2018

Citación: Rodríguez, G. (2018). Caracterización petrográfica, química y edad Ar-Ar de cuerpos porfídicos intrusivos en la formación Saldaña. Boletín Geológico, 44, 5-23.

\section{RESUMEN}

En el valle superior del Magdalena se presentan cuerpos subvolcánicos de andesitas y latitas porfídicas que intruyen las lavas y tobas de la formación Saldaña del Jurásico Inferior. Estos cuerpos se caracterizan por presentar fenocristales de plagioclasa hasta de $2 \mathrm{~cm}$, junto a piroxenos, olivino y sanidina que flotan en una matriz hialocristalina. Corresponden a plutones hipoabisales alcalinos, metaluminosos, con contenidos altos de álcalis, que clasifican químicamente como andesitas. Se interpreta que estas rocas fueron generadas por subducción en un ambiente de arco, con anomalías negativas de $\mathrm{Nb}$ y Ti y a la relación LREE/HREE en los diagramas multielementales. Se obtuvo una edad de meseta en plagioclasa, por el método Ar-Ar, de 159,35 —3,55 Ma, que sugiere, junto con las características químicas y relaciones estratigráficas, que corresponde a un evento magmático más joven que el de las lavas de la formación Saldaña $(186,8 \pm 2,0 \mathrm{Ma}-188,9 \pm 1,6 \mathrm{Ma})$, las vulcanitas de Pitalito (168 $\pm 2,5 \mathrm{Ma}-172,4 \pm 1,7$ $\mathrm{Ma})$ y los plutones jurásicos que afloran en el valle superior del Magdalena (169,4 $\pm 3-195,8 \pm 1,5 \mathrm{Ma})$.

Palabras clave: magmatismo, Jurásico, andesitas, Colombia, geoquímica.

\section{A B S T R A C T}

In the Upper Magdalena Valley, subvolcanic porphyritic andesite and latite rock bodies intrude volcanic lavas and tuffs of the Lower Jurassic Saldana Formation. These rock bodies are characterized by plagioclase phenocrysts up to $2 \mathrm{~cm}$ in diameter, together with pyroxene, olivine and sanidine

Especialista en Evaluación Técnica-Económica de Proyectos Mineros. Servicio Geológico Colombiano, Sede Medellín. 
crystals, floating in a hyalocrystalline matrix. They correspond to hypabyssal, alkaline plutons of the metaluminous series, with high alkali content, chemically classified as andesites and interpreted to be generated by subduction in an arc environment, in accordance with their negative $\mathrm{Nb}$ and $\mathrm{Ti}$ anomalies and LREE/HREE ratios in multielement diagrams. An Ar-Ar plateau age of $159.35 \pm 3.55$ Ma was obtained in plagioclase, which suggests, along with the chemical characteristics and stratigraphic relationships, that it corresponds to a magmatic event younger than the lavas of the Saldana Formation $(186.8 \pm 2.0-188.9 \pm 1.6 \mathrm{Ma})$, the Pitalito Vulcanites $(168 \pm 2.5-172.4 \pm 1.7 \mathrm{Ma})$ and the Jurassic plutons that crop out in the Upper Magdalena Valley $(169.4 \pm 3-195.8 \pm 1.5 \mathrm{Ma})$.

Keywords: Magmatism, Jurassic, andesites, Colombia, geochemistry.

\section{INTRODUCCIÓN}

as rocas ígneas jurásicas de los Andes de Colombia corresponden a cuerpos plutónicos y rocas volcánicas con edades de entre $\sim 214 \mathrm{Ma}$ y $\sim 145 \mathrm{Ma}$, que se distribuyen en bloques tectónicos localizados en la margen oriental de la cordillera Central, el valle del río Magdalena, la cordillera Oriental, la Sierra Nevada de Santa Marta y la alta Guajira (figura 1), estudiadas por diversos autores (Goldsmith et al., 1971; Ward et al., 1973; Álvarez, 1983; Aspden et al., 1987; Gendall et al., 2000; Bustamante et al., 2010, Villagómez et al., 2011; Leal-Mejía, 2011; Van der Lelij, 2013; Mantilla et al., 2013, Van der Lelij et al., 2016; Rodríguez et al., 2017a).

El magmatismo jurásico del norte de los Andes se ha interpretado a partir de dos modelos: desarrollo de un rift intracontinental (Pindell y Dewey, 1982; Mojica et al., 1996; Cediel et al., 2003; Cochrane et al., 2014) y un modelo de magmatismo de arco de margen continental, que es el más aceptado actualmente (McCourt et al., 1984; Toussaint, 1995; Meschede y Frisch, 1998; Bustamante et al., 2010; Leal-Mejía, 2011; Van der Lelij et al., 2016; Spikings et al., 2015; Rodríguez et al., 2015a; Villagómez et al., 2015).

El magmatismo jurásico del valle superior del Magdalena (VSM) comprende batolitos, stocks y unidades volcanosedimentarias espacialmente distribuidos en el borde oriental de la cordillera Central y en el borde occidental de la cordillera Oriental de Colombia. Todos estos cuerpos fueron agrupados de acuerdo a su posición espacial actual, composición y edad, en plutones occidentales y plutones orientales, y muestran concordancia en distribución espacial, composición química y edad con las unidades volcánicas (Rodríguez et al., 2015a, 2018).

La formación Saldaña, en el sentido señalado por Rodríguez et al. (2016), aflora junto a los plutones occiden- tales y presenta edades de cristalización que varían entre $188,9 \pm 4,2$ y $186 \pm 2,0 \mathrm{Ma}$, con algunas edades intermedias alrededor de $183 \mathrm{Ma}$, similares a las arrojadas por el grupo de plutones occidentales. Las vulcanitas de Pitalito se localizan junto a los plutones orientales y presentan edades entre $172,4 \pm 1,7 \mathrm{Ma}$ y $168 \pm 2,5 \mathrm{Ma}$, edades intermedias alrededor de 183-178 Ma, similares a las arrojadas por el grupo de plutones orientales (Rodríguez et al., 2017a).

Los cuerpos de pórfidos objeto de este trabajo corresponden a plutones de poca extensión que afloran junto a la formación Saldaña y a cuerpos intrusivos del Jurásico Inferior, fueron cartografiados y asociados a la formación Saldaña a finales del siglo pasado y en los trabajos de cartografía en la práctica fueron separados, pero no descritos (Fuquen et al., 1989; Rodríguez y Fuquen, 1989, y Carvajal et al., 1993).

En este trabajo se hace una caracterización petrográfica, geoquímica y geocronológica de algunos cuerpos subvolcánicos que intruyen lavas y tobas de la formación Saldaña y plutones del Jurásico Inferior en el área entre Natagaima y Ataco y al occidente de Teruel, en los departamentos del Tolima y el Huila. Se muestran nuevos datos petrográficos (6), geoquímicos (6) y geocronológicos (1) que sugieren un evento magmático posterior al magmatismo de la formación Saldaña, las vulcanitas de Pitalito y los plutones jurásicos del VSM (Rodríguez et al., 2016, 2018).

La información petrográfica y geoquímica presentada en este trabajo junto a un dato geocronológico por el método Ar-Ar pretende aportar nueva información acerca de la evolución magmática jurásica del valle superior del Magdalena y de sus productos litológicos, incluidos datos de cuerpos subvolcánicos denominados informalmente cuerpos de pórfidos andesíticos y dacíticos (Rodríguez y Fuquen, 1989; Carvajal et al., 1993). 


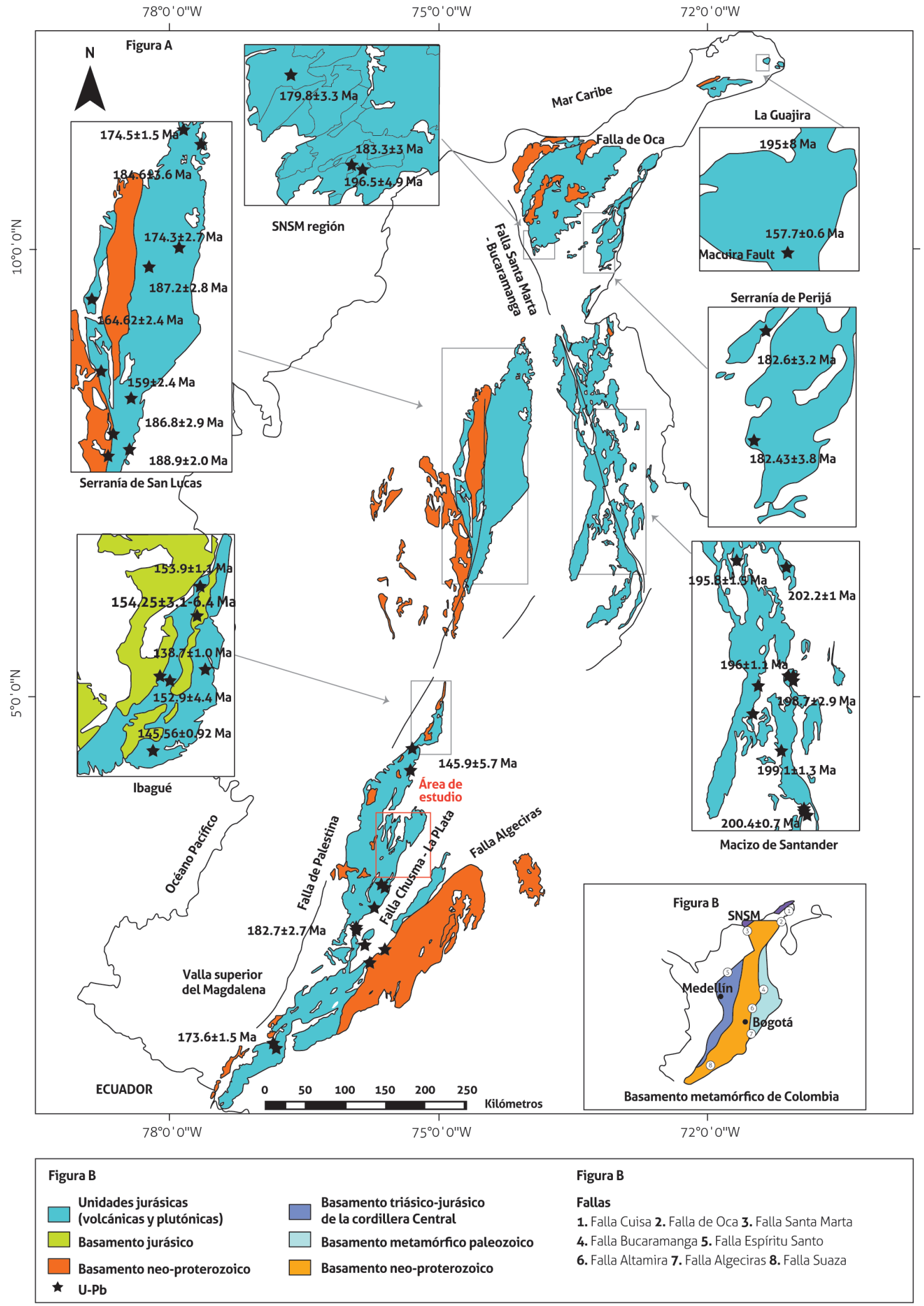

Figura 1. Mapa de localización de afloramientos de rocas ígneas jurásicas en Colombia. El recuadro en rojo señala el área de localización de cuerpos porfídicos

Fuente: modificado de Rodríguez et al. (2018) 


\section{Marco geológico regional}

Desde el punto de vista regional, los cuerpos de pórfido objeto de este trabajo afloran en el valle superior del Magdalena (VSM), sobre las estribaciones orientales de la cordillera Central.

En este sector, el VSM y la cordillera Central presentan un basamento metamórfico del Neoproterozoico (descritos por Kroonenberg y Diederix, 1982; Rodríguez, 1995a, 1995b; Velandia et al., 1996; Velandia et al., 2001, Rodríguez et al., 2003; Jiménez Mejía et al., 2006; Ibáñez-Mejía et al., 2011), sobre el cual reposan rocas sedimentarias paleozoicas (descritas por Stibane y Forero, 1969; Villarroel y Mojica, 1988; Mojica et al., 1988; Velandia et al., 1999; Velandia et al., 2001), y son intruidos por granitoides pérmicos de arco (Rodríguez et al., 2017c). Calizas y sedimentos clásticos del Triásico, como las formaciones Luisa y Payandé, reposan sobre las unidades mencionadas (Geyer, 1973; Cediel et al., 1980; Mojica, 1980).

El basamento neoproterozoico, las formaciones sedimentarias paleozoicas, los granitoides pérmicos y las calizas triásicas fueron intruidos por los plutones jurásicos y cubiertos por el vulcanismo jurásico (Rodríguez et al, 2017c). Los plutones jurásicos del VSM, en la vertiente oriental de la cordillera Central, son calcoalcalinos altos en $\mathrm{K}$, de composición cuarzomonzonítica a monzodiorítica, y aparecen junto a la formación Saldaña, que está constituida por lavas andesíticas, dacíticas, riolíticas, tobas y aglomerados; los plutones y las vulcanitas arrojaron edades U-Pb en circón entre 193 Ma y 186 Ma, principalmente (Rodríguez et al., 2016). Hacia la margen oriental del VSM afloran batolitos y stocks monzograníticos junto a las vulcanitas de Pitalito, que están constituidas por riolitas y tobas; los plutones y las vulcanitas arrojan edades U-Pb en circón de entre $173 \mathrm{Ma}$ y $168 \mathrm{Ma}$ (Rodríguez et al., 2016).

Todo el conjunto de unidades geológicas mencionado está cubierto por secuencias continentales y marinas de rocas sedimentarias de finales del Mesozoico y del Cenozoico, y fueron levantadas a manera de bloques limitados por fallas de rumbo y cabalgamiento, que expusieron en mayor o menor grado las rocas jurásicas y el basamento metamórfico.

Los cuerpos de pórfido hacen parte de un bloque tectónico levantado y limitado al oriente por la falla de Chusma, y al occidente por la falla Inza-Avirama, constituido por rocas pérmicas como el granito de La Plata (Grosse, 1935; Fuquen y Núñez, 1989; Rodríguez, 1995b; Velandia, 2001; Rodríguez et al., 2017c); calizas de la formación Payandé, de edad Triásica Superior (Renz en Trumpy, 1943); rocas volcánicas y piroclásticas de la formación Saldaña del Jurásico Inferior (Cediel et al., 1980, 1981; Rodríguez et al., 2016), junto a plutones cuarzomonzoníticos del Jurásico Inferior, como las cuarzomonzonitas de Anchique (Cossio et al., 1994; Arango et al., 2015); San Cayetano (Carvajal et al., 1993, 1983; Bermúdez et al., 2015) y Los Naranjos (Rodríguez y Fuquen, 1989; Rodríguez et al., 2015b), y sedimentos mesozoicos y cenozoicos, como las formaciones Yavi, Caballos, Hondita, Loma Gorda, La Tabla, Guaduala y el grupo Gualanday, que cubren localmente las unidades triásicas y jurásicas (figura 2). Los cuerpos de pórfidos de composición andesítica intruyen las lavas y rocas piroclásticas de la formación Saldaña y algunos cuerpos plutónicos del Jurásico Inferior. 


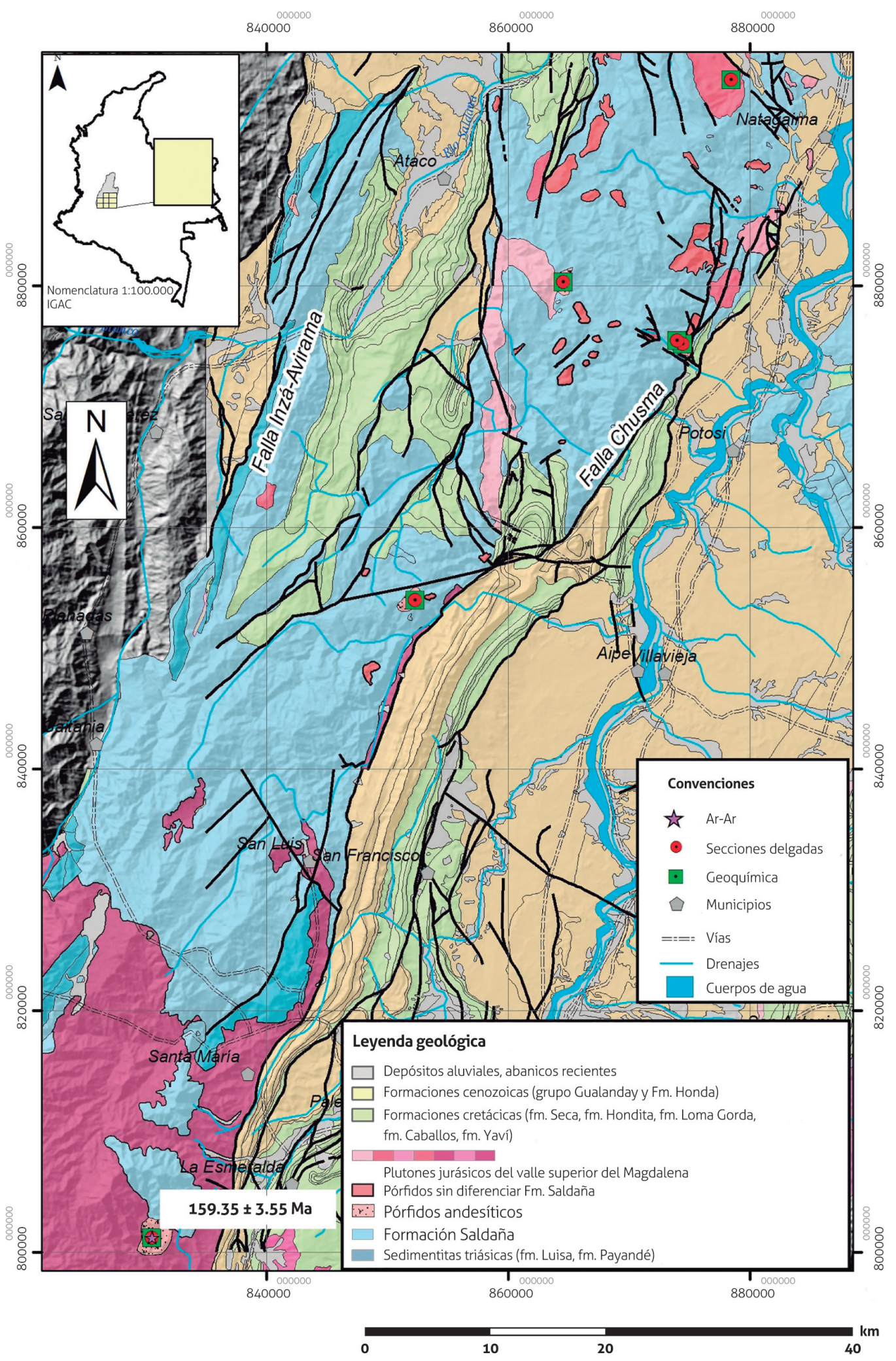

Figura 2. Mapa geológico de las estribaciones orientales de la cordillera Central y el valle superior del Magdalena, en el que se muestra la localización de los sitios de muestreo de andesitas porfídicas

Fuente: modificado de Carvajal et al. (1983); Cossio et al. (1994); Fuquen et al. (1989) y Ferreira et al. (2002) 


\section{TÉCNICAS ANALÍTICAS}

Para el presente estudio se realizaron actividades de compilación de información, control de campo y muestreo, elaboración de secciones delgadas y análisis petrográfico de muestras recolectadas tanto para este estudio como para proyectos anteriores. Utilizando para el análisis petrográfico un microscopio polarizador marca Leitz, se realizó conteo mineralógico a partir de 200 puntos, y para la clasificación se tuvo en cuenta tanto la composición de los fenocristales como de la matriz, clasificando las rocas según el triángulo de Streckeisen (1978). Las secciones delgadas reposan en los laboratorios del Servicio Geológico Colombiano (SGC). Los análisis químicos se realizaron en el laboratorio del Servicio Geológico Colombiano sede Bogotá; los óxidos mayores se determinaron por el método de fluorescencia de rayos $\mathrm{X}$, mediante un equipo Panalytical Axios Mineral, incluyendo los elementos traza $\mathrm{V}, \mathrm{Nb}$ y $\mathrm{Zr}$, y para el resto de elementos de traza y tierras raras se utilizó el equipo de espectrometría de masas con plasma acoplado inductivamente Perkin Elmer NexION (ICP-MS). Para la interpretación de los óxidos mayores se realizó el recálculo teniendo en cuenta que los valores de pérdida por ignición (LOI) fueran menores al $3 \%$.

La cuantificación de los óxidos mayores se realizó en muestra fundida con metaborato y tetraborato de litio, y la cuantificación de elementos menores se realizó en muestra prensada. Para la disolución de la muestra se realizó un ataque por pasos utilizando ácidos inorgánicos fuertes ( $\mathrm{HF}, \mathrm{HNO}_{3}, \mathrm{HClO}_{4}$ y $\mathrm{HCl}$ ). El proceso se realizó en sistema abierto, empleando distintas rampas de temperatura y tiempos de calentamiento.
Se fechó una muestra de andesita porfídica en el OSU Argon Geochronology Lab de la Universidad Estatal de Oregón por el método Ar-Ar, para obtener la edad ${ }^{40} \mathrm{Ar} /{ }^{39} \mathrm{Ar}$ por el método de calentamiento incremental.

De acuerdo con la información suministrada por el laboratorio, la muestra se irradió durante seis horas en el reactor nuclear Triga Clicit, junto con FCT-2 (Fish Canyon Tuff) sanidina $(28,201 \pm 0,023 \mathrm{Ma}, 1 \sigma)$ monitor de flujo (Kuiper et al., 2008). Los valores J individuales se calcularon mediante extrapolación parabólica del gradiente de flujo medido frente a la altura de irradiación y

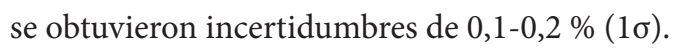

La determinación de la edad de calentamiento incremental ${ }^{40} \mathrm{Ar} /{ }^{39} \mathrm{Ar}$ se realizó en un espectrómetro de masas Argus-VI, con cinco colectores Faraday (todos equipados con resistencias de 1012 ohmios) y un multiplicador de electrones $\mathrm{CuBe}$ con contador de iones (ubicado en una posición al lado del recolector más bajo de faradios). Esto permitió medir simultáneamente todos los isótopos de argón, con la masa 36 en el multiplicador y las masas 37 a 40 en los cuatro faradios adyacentes.

La muestra irradiada se cargó en placa de $\mathrm{Cu}$, en vacío ultraalto y se calentaron gradualmente escaneando un haz láser de $\mathrm{CO}_{2}$ de $25 \mathrm{~W}$ defocus en patrones preestablecidos a lo largo de la muestra, para liberar el argón de manera uniforme. Después del calentamiento se limpiaron los gases reactivos usando un extractor SAES $\mathrm{Zr}$-Al ST101 operado a $400{ }^{\circ} \mathrm{C}$ durante 1,5 a 3 minutos, y dos SAES Fe-V-Zr ST 172 funcionaron a $200^{\circ} \mathrm{C}$ y temperatura ambiente, respectivamente. Antes del análisis, la muestra se horneó a $\sim 125-150{ }^{\circ} \mathrm{C}$, y los gases (reactivos) se bombearon con una bomba turbo.

Tabla 1. Composición modal en muestras de cuerpos de pórfido

\begin{tabular}{|c|c|c|c|c|c|c|c|c|c|c|c|}
\hline \multirow{2}{*}{ N. ${ }^{\circ}$ de campo } & PI & Kfs & Cpx & Орк & ol & Op & $\mathrm{Bt}$ & Tnt & Ap & \multirow{2}{*}{ Matriz } & \multirow{2}{*}{ Clasificación } \\
\hline & \multicolumn{9}{|c|}{ Fenocristales } & & \\
\hline GR-6577 & 9 & & $\mathrm{Tr}$ & & $\mathrm{Tr}$ & 0,7 & & & & 90,3 & Feno andesita \\
\hline GR-6578 & 37,3 & & 1,9 & & 2,5 & 0,6 & & & 0,6 & 57,1 & Andesita \\
\hline MIA-436 & 17 & 7 & 6,5 & & & 1,5 & & & & 68 & Latita \\
\hline JGB-356 & 29,9 & 3,2 & 2,4 & & $\operatorname{Tr}$ & $\operatorname{Tr}$ & & 1,7 & & 62,8 & Andesita \\
\hline GR-6581 & 41,8 & & 3,3 & & 2,5 & 0,8 & & & & 52,5 & Andesita \\
\hline GR-6613 & 25,8 & & 2 & 2,1 & $\mathrm{Tr}$ & 2,9 & 0,7 & $\mathrm{Tr}$ & & 67,1 & Andesita \\
\hline
\end{tabular}

Tr: trazas

Fuente: autor 
El calentamiento incremental se inició alrededor de 1,6-1,8 \% de potencia de láser de $\mathrm{CO}_{2}$ y aumentó por etapas para alcanzar una potencia máxima de láser de $22-24 \%$ de $\mathrm{CO}_{2}$. En total se aplicaron veintitrés etapas de calentamiento incremental para plagioclasa. Se inició con tres medidas de línea base de Faraday, seguidas de un procedimiento en blanco, el último, que luego se repitió una vez cada dos o tres etapas de calentamiento (o fusión) incrementales. Normalmente, se analizaron entre diez y diecisiete espacios en blanco del procedimiento.

La edad se calculó usando la constante de descomposición corregida de Steiger y Jäger (1977) de 5,530 \pm 0,097 x 10-10 1/año (2б), según lo informado por Min et al. (2000). Las edades de meseta de calentamiento incremental y las edades de isócrono se calcularon como medias ponderadas con $1 / \sigma 2$ como factor de ponderación (Taylor, 1997), y como el mínimo cuadrado de York2 se corresponde con errores correlacionados (York, 1969), utilizando el software ArArCALC v2.7.0 de Koppers (2002) disponible en el sitio web http://earthref.org/ArArCALC/.

La reproducibilidad de los análisis de sanidina de Alder Creek (AC-2) es excelente, con edades medias de $1176,3 \pm 3,9$ ka y $1184,0 \pm 3,9$ ka (error interno, $2 \sigma$; MSWD $=5,68 ; n=132 / 143$ ) frente a las edades de sanidina FCT de 28,02 Ma y 28.201 Ma de Kuiper et al. (2008).

\subsection{Resultados}

Descripción macroscópica y microscópica. Los cuerpos de pórfido están constituidos por andesitas y latitas de color gris verdoso moteado de blanco, y rosado moteado de verde claro. Presentan textura porfídica y matriz microcristalina felsítica microgranular o microlítica, con vidrio isotrópico de color verde en algunas muestras. Las muestras están constituidas por fenocristales de plagioclasa de tamaños que oscilan entre 1 y $2 \mathrm{~cm}$, idiomórficos a subidiomórficos; la sanidina puede estar en algunas rocas y son frecuentes piroxenos y olivino con tamaños de 2 a $4 \mathrm{~mm}$; como accesorios se presentan opacos, apatito y en algunas muestras titanita y circón (tabla 1).

La plagioclasa, en algunos casos, tiene una tonalidad rosada, manchada por óxidos de hierro o verde debido a alteración a saussurita y sericita. La matriz es afanítica, de colores gris, gris verdoso y rosado, hialocristalina a felsítica microgranular; ocasionalmente se observan amígdalas blancas rellenas con calcedonia, calcita (figura 3), y pueden tener cobre nativo.
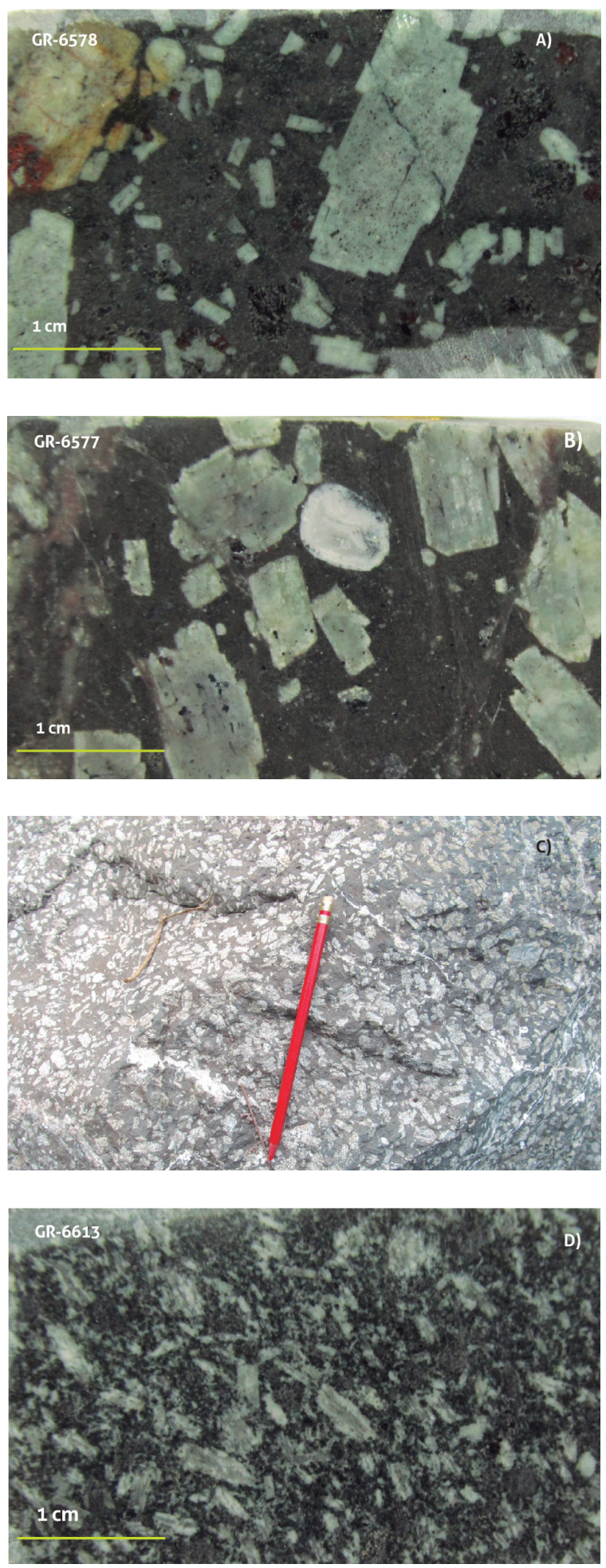

Figura 3. Aspecto macroscópico de rocas de los cuerpos de pórfido andesítico al oeste de Natagaima (Tolima) y Teruel (Huila). A) Muestra GR-6578-andesita con fenocristales de plagioclasa bimodales. B) Muestra GR-6577-andesita con fenocristales de plagioclasa y amígdalas calcita-cuarzo. C) afloramiento de pórfido andesítico. D) muestra GR-6613-andesita con fenocristales de plagioclasa ligeramente orientados

Fuente: autor 
La plagioclasa es de tipo andesina, con composición que varía entre $A_{32}$ y $A_{36}$, se encuentra en fenocristales y como microcristales en la matriz. Los fenocristales son euédricos a subédricos tabulares, de tamaños que oscilan entre 1 y $2 \mathrm{~cm}$, aunque se presentan algunos de menor tamaño (1 a $2 \mathrm{~mm}$ ), con bordes corroídos por la matriz, maclados según la ley de albita, Carlsbad y albita-Carlsbad, y relieve mayor al del bálsamo; se encuentran empolvados por alteración a sericita, arcilla y saussurita, y tienen inclusiones de opacos, pasta de la matriz y olivino serpentinizado (figura 4). Los microcristales en la matriz son anédricos, mal desarrollados, empolvados por alteración a arcilla y sericita que le imprimen el aspecto sucio a la matriz.
La sanidina ocurre en algunos cuerpos de pórfido, generalmente en fenocristales euédricos de $1 \mathrm{a} 2 \mathrm{~cm}$, o como coronas alrededor de fenocristales de plagioclasa y en microcristales en la matriz. Generalmente alterados a arcilla parda y en las coronas con desmezcla pertítica a manera de parches (figura 4).

El clinopiroxeno es augita que se encuentra en microfenocristales y en microcristales en la matriz. Los fenocristales son euédricos a anédricos, de menor tamaño que la plagioclasa $(0,2$ a $0,6 \mathrm{~mm})$, incoloros, con relieve alto $(n>b)$; el color máximo de birrefringencia es el azul del segundo orden; presenta microfracturas irregulares y contornos de los cristales corroídos por la matriz.



Figura 4. Aspecto microscópico de cuerpos de pórfido andesíticos. A) y B) GR-6578-andesita porfídica. Fenocristales euédricos de plagioclasa (Pl), clinopiroxeno (Cpx) y opacos (Op) flotando en una matriz hialocristalina (M). C) MIA-436-latita. Fenocristales de plagioclasa (PI) con bordes de sanidina pertítica (Kfs) y matriz felsítica microgranular (M). D) JGB 356-andesita. Fenocristales de plagioclasa (Pl) con macla de albita junto a fenocristal de clinopiroxeno (Cpx) flotando en matriz hialocristalina (M)

Fuente: autor 
Los microcristales de la matriz se presentan en agregados anédricos mal desarrollados, de color pardo amarillento (figura 4).

Pueden presentar ortopiroxeno en cristales finos euédricos a subédricos en la matriz, con pleocroísmo débil de amarillo pálido a rosado pálido y birrefringencia baja en tonos de gris del primer orden, con fracturamiento transversal irregular y alteración a lo largo de las fracturas a esmectita y talco; puede estar intercrecido con el clinopiroxeno.

Algunas rocas tienen microfenocristales de olivino totalmente alterados a serpentina y a minerales arcillosos, que conservan la forma esquelética de los cristales; son parcialmente reabsorbidos por la matriz, de color verde y tamaños que oscilan entre 0,2 y $0,7 \mathrm{~mm}$, con inclusiones de opacos y apatito, así como fracturas concoideas con magnetita a lo largo de las fracturas o completamente reemplazados por opacos.

Los opacos se encuentran en cristales finos anédricos diseminados en la matriz de la roca y como escasos microfenocristales. Los abundantes microcristales de opacos en la matriz, con tamaños menores de $0,02 \mathrm{~mm}$, le imprimen un aspecto moteado fino a la pasta de la roca (figura 4). El tamaño de los microfenocristales oscila entre 0,1 y $0,3 \mathrm{~mm}$, y pueden estar incluidos en los fenocristales de piroxeno.

Se presentan amígdalas cuyo tamaño oscila entre 2 y $5 \mathrm{~mm}$, de formas ovaladas a irregulares, con estructura interna concéntrica; hacia los bordes tienen bandeo de capas de carbonatos, y en el núcleo, cuarzo en cristales drusiformes.

Geoquímica. Se realizó el análisis geoquímico de seis muestras de roca pertenecientes a cuerpos de pórfidos diferentes, pero con composición y aspecto macroscópico similar, clasificadas petrográficamente como andesitas y latitas. Se comparan con lavas de la formación Saldaña, cuyos resultados químicos fueron tomados de Rodríguez et al. (2016). Los resultados de pórfidos se presentan en la tabla 2, y la localización, en la figura 2. Las muestras de los cuerpos de pórfido presentan contenidos $\mathrm{de}^{\mathrm{SiO}_{2}}$ entre $56,4 \%$ y $60 \%$, valores de $\mathrm{Al}_{2} \mathrm{O}_{3}$ altos entre $16,5 \%$ y $19 \%$; valores de $\mathrm{CaO}$ entre $2,4 \%$ y $6,6 \%$; valores altos de $\mathrm{Na}_{2} \mathrm{O}$ de $2,9 \%$ a $5,1 \%$; $\mathrm{K}_{2} \mathrm{O}$ entre $2,8 \%$ y $5,3 \%$; los contenidos de $\mathrm{Fe}_{2} \mathrm{O}_{3}$ son similares en todas las rocas: entre $5,3 \%$ y $7,0 \%$; los valores de $\mathrm{P}_{2} \mathrm{O}_{5}$, entre $0,4 \%$ y $0,5 \%$; contenidos de $\mathrm{TiO}_{2}<1 \%$, excepto en la muestra GR-6613, con un contenido de 1,02\%. La mayoría de muestras de la formación Saldaña presenta mayores contenidos $\mathrm{de}_{\mathrm{SiO}_{2}}$, con valores mayores al $60 \%$.

Las muestras de los pórfidos (en rojo) grafican en el diagrama de Le Bas et al. (1986) en el campo de las andesitas y traquiandesitas, cuatro de ellas en la serie alcalina, excepto las muestras GR-6613 y GR-6581, que se grafican en el campo subalcalino (figura 5). Las rocas presentan valores de $\mathrm{Na}_{2} \mathrm{O}-2,0<\mathrm{K}_{2} \mathrm{O}$, que permite subdividirlas y clasificarlas químicamente como latitas cercanas al campo de las shoshonitas, de acuerdo a Le Maitre et al. (2002). El álcalis $\left(\mathrm{Na}_{2} \mathrm{O}+\mathrm{K}_{2} \mathrm{O}\right)$ generalmente es móvil en rocas alteradas, que presentan moderada alteración de la plagioclasa a saussurita y arcilla.

El diagrama de Winchester y Floyd (1977) de Zr/

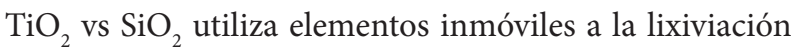
por alteración. Las rocas se grafican en el campo de las andesitas subalcalinas, cerca del límite que las separa de las traquiandesitas alcalinas (figura 5). Al comparar con muestras de lavas de la formación Saldaña (en negro), que caen en la serie subalcalina, se observa agrupación y separación de las muestras de pórfidos en los diagramas

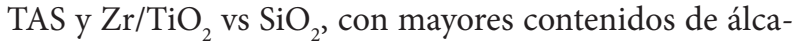
lis, $\mathrm{K}_{2} \mathrm{O}$ y $\mathrm{Zr} / \mathrm{TiO}_{2}$ para los pórfidos a valores similares de $\mathrm{SiO}_{2}$. La clasificación petrográfica de la mayoría de rocas se localiza en el campo de las andesitas del triángulo de Streckeisen (1978). Químicamente se localizan en el diagrama TAS en los campos de las andesitas (1), traquiandesitas (4) y traquidacitas (1), y en el diagrama de $\mathrm{Zr}$ / $\mathrm{TiO}_{2} \mathrm{vs} \mathrm{SiO}_{2}$ caen en el campo de las andesitas, y se clasifican de manera similar. 
Rodríguez García

Tabla 2. Contenido de óxidos mayores y elementos trazas en muestras de pórfidos de la formación Saldaña

\begin{tabular}{|c|c|c|c|c|c|c|}
\hline Muestra & GR-6581 & GR-6577 & GR-6578 & MIA-436 & GR-6613 & JGB-356 \\
\hline Nombre petrográfico & Andesita & Andesita & Andesita & Latita & Andesita & Andesita \\
\hline $\mathrm{N}$ & 880327 & 875174 & 875441 & 853985 & 801186 & 897068 \\
\hline w & 864583 & 874439 & 874009 & 852317 & 830525 & 878480 \\
\hline $\mathrm{SiO}_{2}$ & 56,61 & 58,67 & 56,37 & 59,99 & 56,58 & 55,93 \\
\hline $\mathrm{TiO}_{2}$ & 0,95 & 0,73 & 0,94 & 0,94 & 1,03 & 0,870 \\
\hline $\mathrm{Al}_{2} \mathrm{O}_{3}$ & 17,39 & 18,11 & 17,69 & 16,47 & 16,41 & 19,00 \\
\hline $\mathrm{Fe}_{2} \mathrm{O}_{3}$ & 6,84 & 5,29 & 6,69 & 6,01 & 7,97 & 6,07 \\
\hline $\mathrm{MgO}$ & 2,55 & 1,81 & 2,37 & 1,64 & 3,88 & 1,87 \\
\hline $\mathrm{CaO}$ & 6,31 & 4,01 & 5,78 & 2,40 & 6,59 & 5,36 \\
\hline $\mathrm{Na}_{2} \mathrm{O}$ & 3,08 & 5,06 & 3,82 & 4,66 & 2,91 & 3,49 \\
\hline $\mathrm{K}_{2} \mathrm{O}$ & 3,80 & 3,45 & 3,68 & 5,33 & 2,80 & 4,68 \\
\hline $\mathrm{P}_{2} \mathrm{O}_{5}$ & 0,43 & 0,48 & 0,50 & 0,43 & 0,38 & 0,54 \\
\hline $\mathrm{MnO}$ & 0,01 & 0,01 & 0,01 & 0,01 & 0,01 & 0,01 \\
\hline $\mathrm{FeO}$ & 3,13 & 2,70 & 3,29 & 1,68 & 5,84 & 1,72 \\
\hline LOI & 1,60 & 1,94 & 1,74 & 1,72 & 1,11 & 2,31 \\
\hline Li & 15,66 & 18,02 & 26,48 & 24,46 & 10,56 & 24,74 \\
\hline $\mathrm{Be}$ & 1,61 & 2,17 & 2,04 & 2,81 & 2,64 & 2,07 \\
\hline Sc & 17,91 & 11,05 & 15,26 & 13,18 & 27,54 & 15,01 \\
\hline$V^{*}$ & 123,24 & 95,23 & 134,44 & 100,83 & 156,85 & 114,20 \\
\hline $\mathrm{Cr}$ & 28,81 & 11,08 & 15,63 & 14,70 & 49,84 & 12,00 \\
\hline Co & 19,27 & 13,94 & 17,15 & 13,03 & 26,28 & 15,65 \\
\hline $\mathrm{Ni}$ & 16,55 & 9,01 & 13,56 & 9,15 & 28,21 & 10,25 \\
\hline $\mathrm{Cu}$ & 74,46 & 129,72 & 217,35 & 85,35 & 54,20 & 79,96 \\
\hline $\mathrm{Zn}$ & 88,23 & 74,18 & 80,83 & 93,65 & 125,28 & 84,94 \\
\hline Ga & 19,13 & 20,46 & 21,77 & 22,52 & 20,78 & 21,31 \\
\hline As & 2,75 & 2,29 & 1,63 & 3,10 & 1,03 & 4,92 \\
\hline $\mathrm{Rb}$ & 136,87 & 90,49 & 122,50 & 177,06 & 104,59 & 126,37 \\
\hline $\mathrm{Sr}$ & 565,29 & 928,37 & 1372,66 & 609,49 & 571,32 & 1086,89 \\
\hline Y & 28,76 & 21,10 & 24,06 & 31,80 & 28,59 & 24,50 \\
\hline $\mathrm{Zr}^{*}$ & 244,30 & 192,48 & 199,88 & 281,31 & 244,30 & 219,40 \\
\hline $\mathrm{Nb}^{*}$ & 7,80 & 6,40 & 6,90 & 10,90 & 9,60 & 7,00 \\
\hline Cs & 1,20 & 2,15 & 1,35 & 3,55 & 3,19 & 0,99 \\
\hline $\mathrm{Ba}$ & 1080,68 & 1404,52 & 1391,07 & 1823,82 & 924,87 & 1518,59 \\
\hline La & 29,84 & 40,16 & 40,03 & 62,38 & 40,47 & 41,17 \\
\hline $\mathrm{Ce}$ & 66,84 & 79,14 & 79,24 & 123,85 & 80,92 & 74,04 \\
\hline $\mathrm{Pr}$ & 7,93 & 9,20 & 9,48 & 14,19 & 10,32 & 10,01 \\
\hline $\mathrm{Nd}$ & 32,02 & 37,95 & 38,90 & 53,82 & 39,54 & 40,04 \\
\hline Sm & 7,17 & 7,49 & 7,78 & 10,74 & 8,70 & 8,34 \\
\hline Eu & 2,20 & 2,46 & 2,67 & 3,34 & 2,65 & 2,67 \\
\hline Gd & 5,34 & 5,37 & 5,88 & 8,50 & 6,52 & 7,20 \\
\hline $\mathrm{Tb}$ & 1,00 & 0,94 & 1,02 & 1,38 & 1,20 & 1,03 \\
\hline Dy & 5,16 & 4,40 & 4,99 & 6,45 & 5,73 & 5,26 \\
\hline Ho & 1,04 & 0,84 & 0,96 & 1,28 & 1,12 & 1,12 \\
\hline Er & 3,14 & 2,50 & 2,90 & 4,09 & 3,34 & 3,32 \\
\hline $\mathrm{Tm}$ & 0,42 & 0,32 & 0,37 & 0,54 & 0,44 & 0,45 \\
\hline $\mathrm{Yb}$ & 2,85 & 2,06 & 2,42 & 3,57 & 2,94 & 2,98 \\
\hline Lu & 0,46 & 0,31 & 0,36 & 0,55 & 0,45 & 0,47 \\
\hline $\mathrm{Tl}$ & 0,34 & 0,18 & 0,13 & 0,32 & 0,52 & 0,25 \\
\hline $\mathrm{Pb}$ & 9,37 & 12,59 & 14,04 & 19,84 & 13,73 & 15,78 \\
\hline Th & 7,18 & 7,44 & 8,66 & 15,31 & 11,18 & 7,55 \\
\hline U & 1,85 & 1,84 & 2,12 & 3,49 & 3,11 & 2,07 \\
\hline
\end{tabular}

* Elementos traza analizados por FRX

Fuente: autor 
En el diagrama de $\mathrm{SiO}_{2}$ vs $\mathrm{K}_{2} \mathrm{O}$, las muestras presentan alta dispersión, probablemente debida a movilidad causada por alteración; cuatro muestras se grafican en la serie shoshonítica (GR-6581, GR-6578, MIA-436 y JGB356), y dos (GR-6577 y GR-6613) en el campo de la serie calcoalcalina alta en $\mathrm{K}$; tienen alto contenido de álcalis $\left(\mathrm{Na}_{2} \mathrm{O}+\mathrm{K}_{2} \mathrm{O}>5 \%\right)$ entre $5,7 \%$ y $10 \%$, alta relación
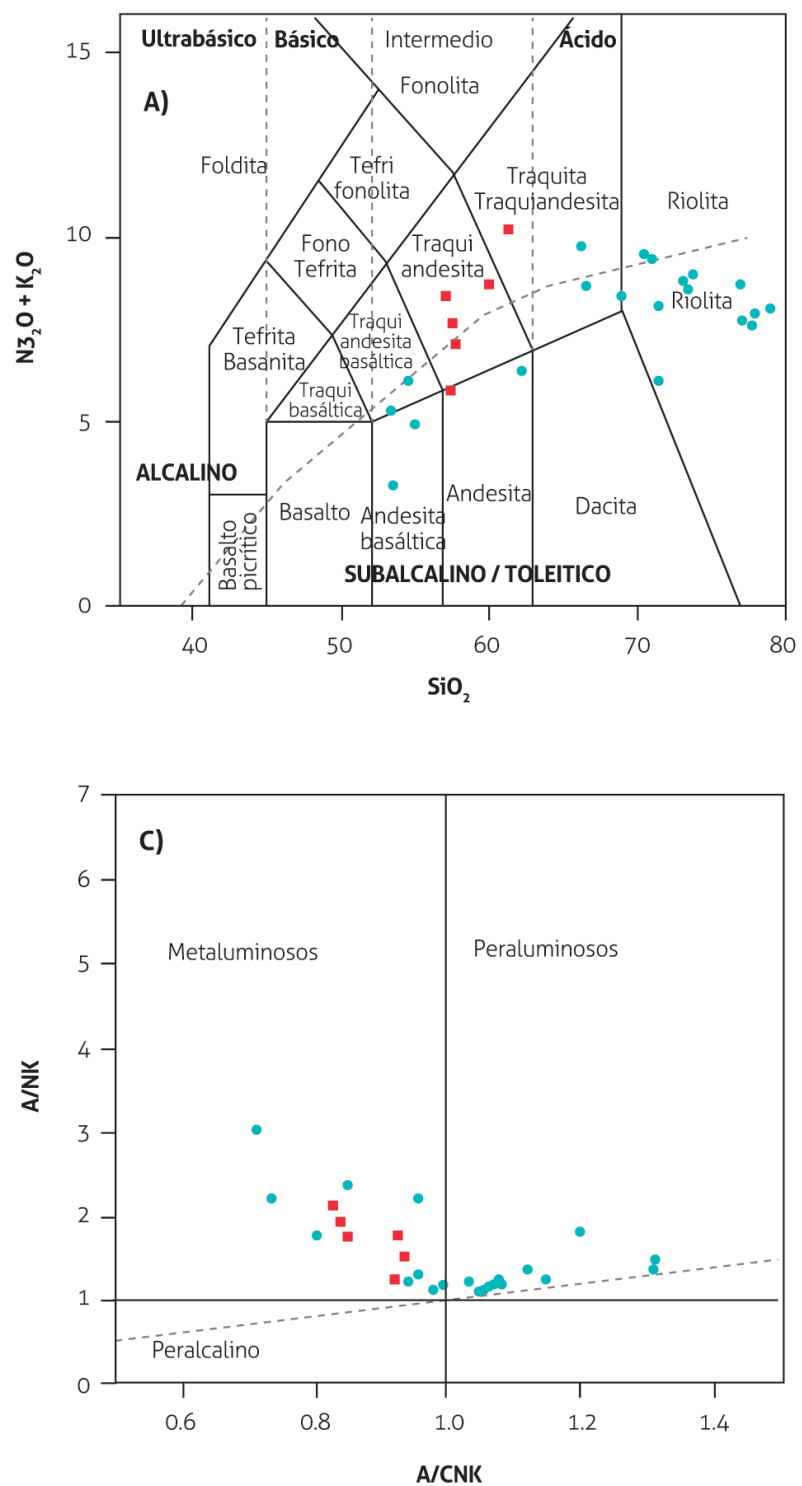

$\mathrm{K}_{2} \mathrm{O} / \mathrm{Na}_{2} \mathrm{O}$ entre $0,68 \%$ y $1,3 \%$, y valores altos de $\mathrm{Al}_{2} \mathrm{O}_{3}$, afines con los valores de la serie shoshonítica (figura 5). Las rocas son metaluminosas, con relación $\mathrm{A} / \mathrm{CNK}<1$, valores de $\mathrm{A} / \mathrm{NK}$ que oscilan entre 1 y 2,2. Las lavas de la formación Saldaña (en azul) muestran alta dispersión, la mayoría están en la serie calcoalcalina, y la mayor parte de las muestras son peraluminosas.
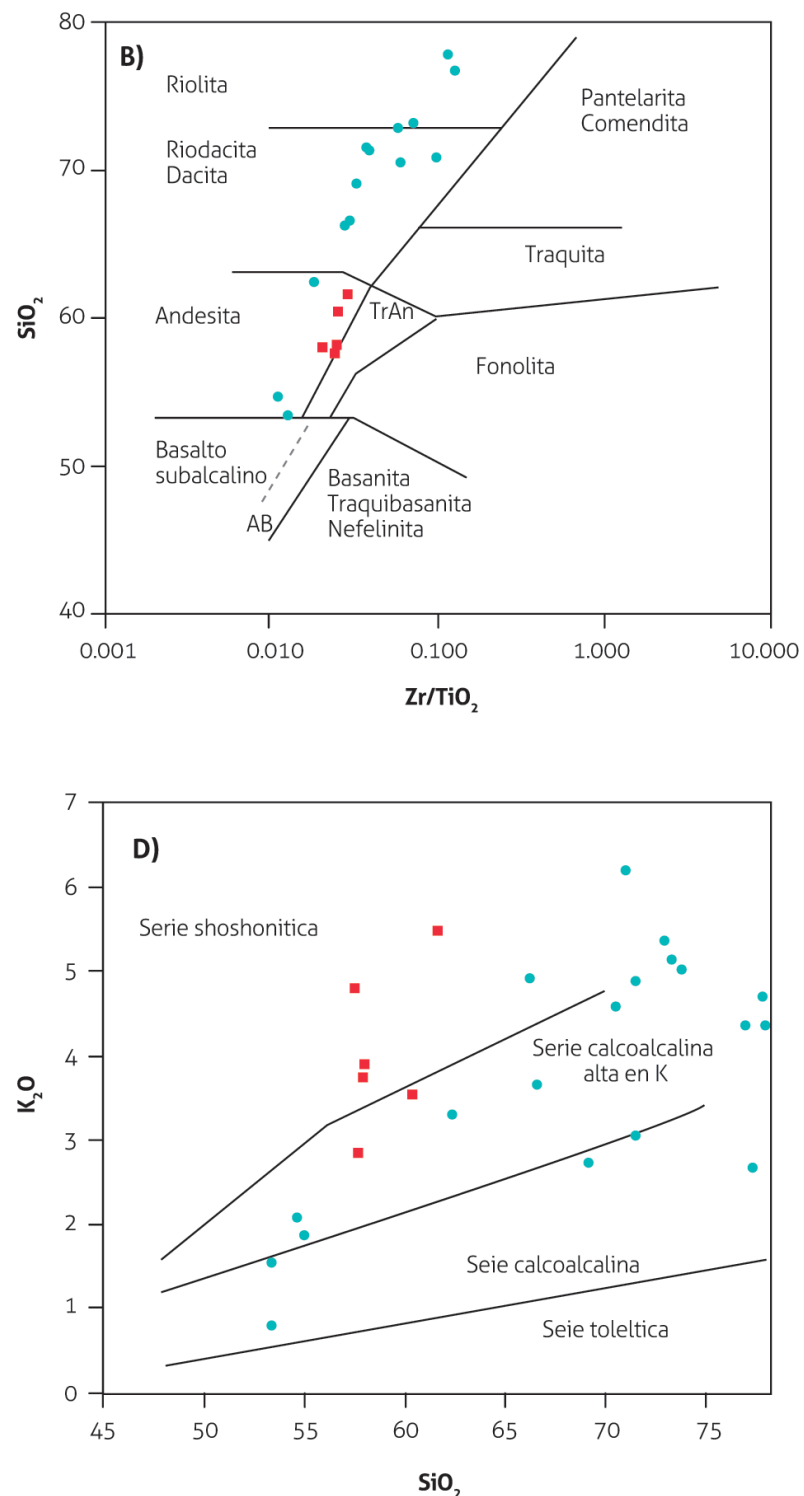

Figura 5. Muestras de cuerpos de pórfidos (en rojo) y lavas de la formación Saldaña (en azul). A) Diagrama de álcalis vs SiO (TAS; Le Bas et al., 1986). B) Diagrama de clasificación $\mathrm{Zr} / \mathrm{TiO}_{2}$ vs $\mathrm{SiO}_{2}$ (Winchester y Floyd, 1977). C) Diagrama de alcalinidad-aluminosidad (Shand, 1943). D) Diagrama de $\mathrm{K}_{2} \mathrm{O}$ vs $\mathrm{SiO}_{2}$ (Peccerillo y Taylor, 1976) 
Los elementos traza fueron normalizados con respecto a N-MORB (basaltos de dorsal oceánica normales que representan el manto empobrecido en elementos incompatibles por procesos de fusión parcial). Las muestras de pórfidos presentan marcada anomalía negativa de $\mathrm{Nb}$ y $\mathrm{Ti}$, que puede deberse a separación de fases minerales como titanita y rutilo (Winter, 2001), donde el Nb y Ti se comportan de manera similar; además, presentan anomalía negativa de $\mathrm{Nb}$ con respecto al Th y Ce, signatura geoquímica típica de magmas originados en ambientes tectónicos relacionados con arcos (Pearce, 1996), con patrón subparalelo para las muestras. Presenta anomalía positiva y valores altos de $\mathrm{Cs}, \mathrm{Rb}, \mathrm{Ba}$, Th, $\mathrm{Sr}, \mathrm{K}, \mathrm{y} \mathrm{Pb}$; característica de ambientes de arco magmáticos de margen continental (Pearce et al., 1984; Pearce, 1996), con empobrecimiento progresivo de los large ion lithophile elements (LILE) hacia high field strenght (HFSE), y una

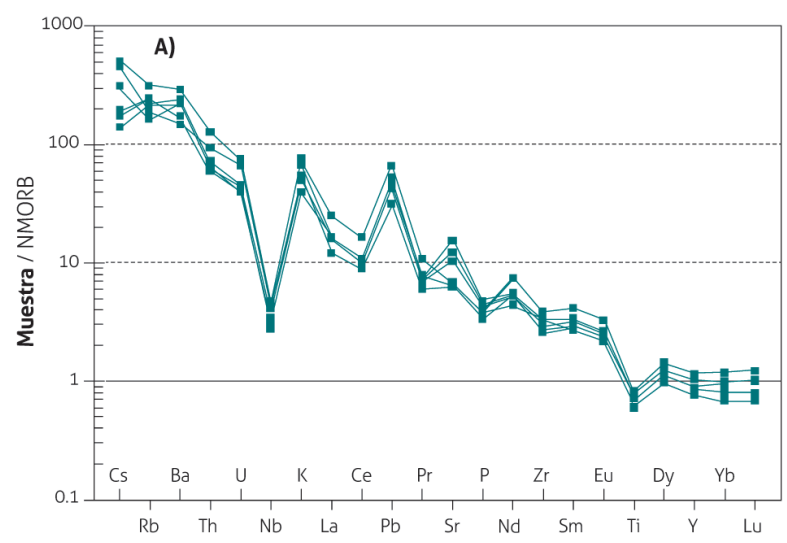

alta relación entre HFSE/LILE que sugiere un origen para estos cuerpos relacionado a subducción en un ambiente de arco de margen continental (figura 6A).

Los patrones de tierras raras (rare earth elements [REE]) normalizados al condrito de Nakamura (1974) son paralelos, con pendiente negativa y patrones comparables al de rocas generadas en ambientes de subducción por encima de la placa subducida, con enriquecimiento en tierras raras livianas (light rare earth elements [LREE]) y empobrecimiento hacia las tierras raras pesadas (heavy rare earth elements [HREE]) (figura 6B); la relación (La/ $\mathrm{Yb})_{\mathrm{N}}$ varía entre 7 y 12, lo cual sugiere aporte cortical, al igual que la relación $(\mathrm{La} / \mathrm{Sm})_{\mathrm{N}}$, que varía entre 2,6 y 3,6 (tabla 3). Presentan leve anomalía positiva de Eu, con relaciones $\mathrm{Eu} / \mathrm{Eu}^{*}>1$ entre 1,07 y $1,21\left(\mathrm{Eu} / \mathrm{Eu}^{*}=\mathrm{Eu}_{\mathrm{N}} /\right.$ $\left(\left(\mathrm{Sm}_{\mathrm{N}}\right) \mathrm{x}\left(\mathrm{Gd}_{\mathrm{N}}\right)^{1 / 2}\right)$ que se atribuye a que no se dio fraccionamiento de la plagioclasa.

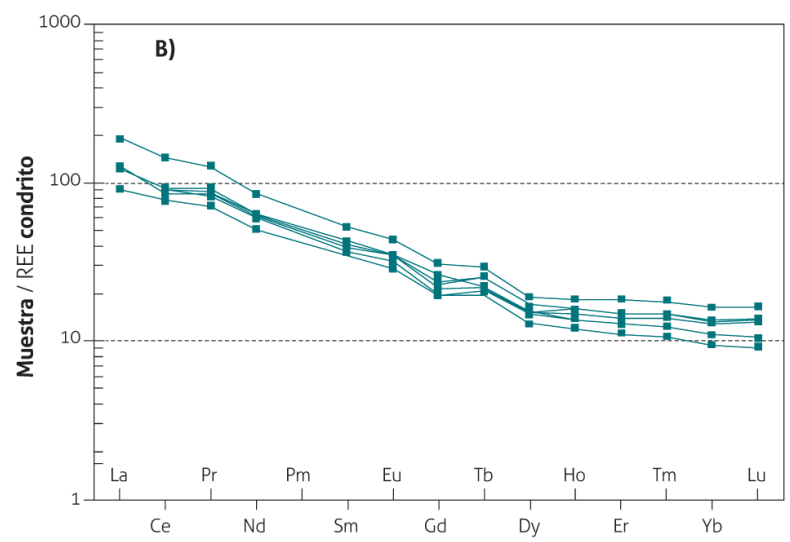

Figura 6. Diagramas multielementales de elementos traza y tierras raras para rocas de cuerpos porfídicos. A) Diagrama de elementos traza normalizado respecto a N-MORB (Sun y Mcdonough, 1989). B) Diagrama de elementos de las tierras raras normalizado respecto al condrito (Nakamura, 1974)

Fuente: autor

Tabla 3. Valores normalizados de REE según el condrito de Nakamura (1974) para muestras de rocas de los pórfidos andesíticos

\begin{tabular}{|c|c|c|c|c|c|c|}
\hline Muestra & $\mathrm{Eu} / \mathrm{Eu}^{*}$ & $(\mathrm{La} / \mathrm{Yb}) \mathrm{N}$ & (La/Sm)N & $(\mathrm{Ce} / \mathrm{Yb}) \mathrm{N}$ & $(\mathrm{Ce} / \mathrm{Sm}) \mathrm{N}$ & $(\mathrm{Eu} / \mathrm{Yb}) \mathrm{N}$ \\
\hline GR-6581 & 1,09 & 6,99 & 2,56 & 5,97 & 2,19 & 2,21 \\
\hline GR-6577 & 1,19 & 12,97 & 3,30 & 9,75 & 2,48 & 3,40 \\
\hline GR-6578 & 1,21 & 11,03 & 3,17 & 8,33 & 2,39 & 3,15 \\
\hline MIA-436 & 1,07 & 11,66 & 3,57 & 8,83 & 2,71 & 2,67 \\
\hline GR-6613 & 1,08 & 9,18 & 2,86 & 7,00 & 2,18 & 2,57 \\
\hline JGB-356 & 1,06 & 9.21 & 3,04 & 6,32 & 2.08 & 2,56 \\
\hline
\end{tabular}

Fuente: autor 
Al graficar las rocas en los diagramas de discriminación de ambiente tectónico propuestos por Wood (1980), las muestras se localizan en el campo de arco volcánico calcoalcalino (figura 7), concordando con el ambiente de la formación Saldaña (azul).

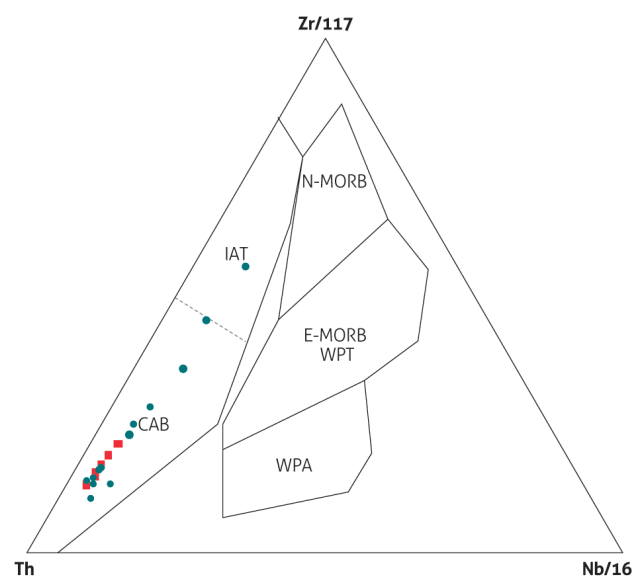

Figura 7. Diagrama de discriminación geoquímica y ambiente tectónico para cuerpos porfídicos del VSM; diagrama de Wood (1980). (WPA: basaltos alcalinos de intraplaca, IAT: arcos de isla toleiticos; $\mathrm{CAB}$ : basaltos de arco calcoalcalino). En rojo, muestras de pórfidos, y en negro, lavas de la formación Saldaña

Fuente: autor
Geocronología. En los cuerpos de pórfidos se dataron tres muestras de roca por el método $\mathrm{Ar} / \mathrm{Ar}$ con calentamiento por pasos, sin resultados en dos de ellas, en las que se utilizó para la datación la matriz y roca total (GR6577 y GR6581). Se obtuvo para la muestra GR-6613 un espectro de edad discordante, con dieciséis pasos, que arrojó una edad de meseta (plateau) en plagioclasa de $159,35 \pm 0,44 \mathrm{Ma}(\mathrm{n}=7)$, que se caracteriza por el $32 \%$ de ${ }^{39}$ Ar liberado (figura 8). En la tabla 4 se resumen los datos emitidos por el laboratorio de la Universidad de Oregón, donde se muestran edades similares a la edad Plateau, en la isócrona inversa y en la edad de fusión total. Esta última sería la edad mínima cuando la estimación o ubicación de la meseta no es clara, como es el caso de esta muestra.

La muestra presenta edades de meseta, isócrona y fusión total similar, entre 158 y $161 \mathrm{Ma}$ (tabla 4), rango en el que se encuentra el valor de la edad Ar-Ar de cierre de la plagioclasa. El espectro de edad es creciente, como se observa en la figura 8 , donde los pasos de la meseta son ligeramente crecientes alrededor de $160 \mathrm{Ma}$ en dieciséis de los veintinueve pasos.

Tabla 4. Resumen de la datación Ar-Ar de la muestra GR-6613 reportados por el Laboratorio de la Universidad de Oregón

\begin{tabular}{|c|c|c|c|c|c|c|c|c|c|c|}
\hline \multicolumn{3}{|c|}{ Resultados } & \multicolumn{8}{|c|}{ Plateau } \\
\hline Muestra & Tipo & Mineral & Edad $\pm 2 \sigma(i)$ & $\pm 2 \sigma(f)$ & ${ }^{39} \mathrm{Ar}$ & $\mathrm{K} / \mathrm{Ca} \pm 2$ & MSWD & $\mathbf{P}$ & $\mathbf{n}$ & $\mathbf{N}$ \\
\hline GR-6613 & Calentamiento incremental & Plagioclase & $159,35 \pm 0,44 \mathrm{Ma}$ & $\pm 3,55 \mathrm{Ma}$ & $32 \%$ & $0,092 \pm 0,00$ & 4,9 & $0 \%$ & 7 & 29 \\
\hline \multicolumn{11}{|c|}{ Isócrona normal } \\
\hline \multicolumn{2}{|r|}{ Edad $\pm 2 \sigma(i)$} & \multicolumn{2}{|c|}{ $\pm 2 \sigma(f)$} & \multicolumn{2}{|c|}{${ }^{40} \mathrm{Ar} /{ }^{36} \mathrm{Ar} \pm 2 \sigma$ - intercepto } & \multicolumn{2}{|c|}{ MSWD } & \multicolumn{3}{|c|}{$\mathbf{P}$} \\
\hline \multicolumn{2}{|r|}{$160,8 \pm 0,71 \mathrm{Ma}$} & \multicolumn{2}{|c|}{ $\pm 3,62 \mathrm{Ma}$} & \multicolumn{2}{|c|}{$268,09 \pm 12,47$} & \multicolumn{2}{|c|}{1,18} & \multicolumn{3}{|c|}{$31 \%$} \\
\hline \multicolumn{11}{|c|}{ Fusión total } \\
\hline \multicolumn{3}{|c|}{ Edad $\pm 2 \sigma(i)$} & \multicolumn{3}{|c|}{ $\pm 2 \sigma(f)$} & \multicolumn{5}{|c|}{$\mathrm{K} / \mathrm{Ca} \pm 2 \sigma$} \\
\hline \multicolumn{3}{|c|}{$158,25 \pm 0,24 \mathrm{Ma}$} & \multicolumn{3}{|c|}{ $\pm 3,50 \mathrm{Ma}$} & \multicolumn{5}{|c|}{$0,089 \pm 0$} \\
\hline \multicolumn{11}{|c|}{ Isócrona inversa } \\
\hline \multicolumn{2}{|r|}{ Edad $\pm 2 \sigma(i)$} & $\pm 2 \sigma(f)$ & \multicolumn{2}{|c|}{${ }^{40} \mathrm{Ar} /{ }^{36} \mathrm{Ar} \pm 2 \sigma$-intercepto } & SF & \multicolumn{2}{|c|}{ MSWD } & \multicolumn{3}{|c|}{$\mathbf{P}$} \\
\hline \multicolumn{2}{|r|}{$160,81 \pm 0,72 \mathrm{Ma}$} & $\pm 3,62 \mathrm{Ma}$ & \multicolumn{2}{|c|}{$267,81 \pm 12,48$} & $7 \%$ & \multicolumn{2}{|c|}{1,19} & \multicolumn{3}{|c|}{$31 \%$} \\
\hline
\end{tabular}

Fuente: autor

\section{DisCUSIÓN Y CONCLUSIONES}

Las muestras analizadas corresponden a diferentes cuerpos que intruyen tanto la formación Saldaña como los plutones de edad Jurásico Inferior del VSM. La similitud macroscópica y microscópica en composición y textura sugiere condiciones de enfriamiento y cristalización similares, rápidas, de acuerdo a las texturas microcristalinas y hialocristalinas microlíticas, lo cual a su vez sugiere niveles de emplazamiento subvolcánicos comparables para todos los cuerpos. 

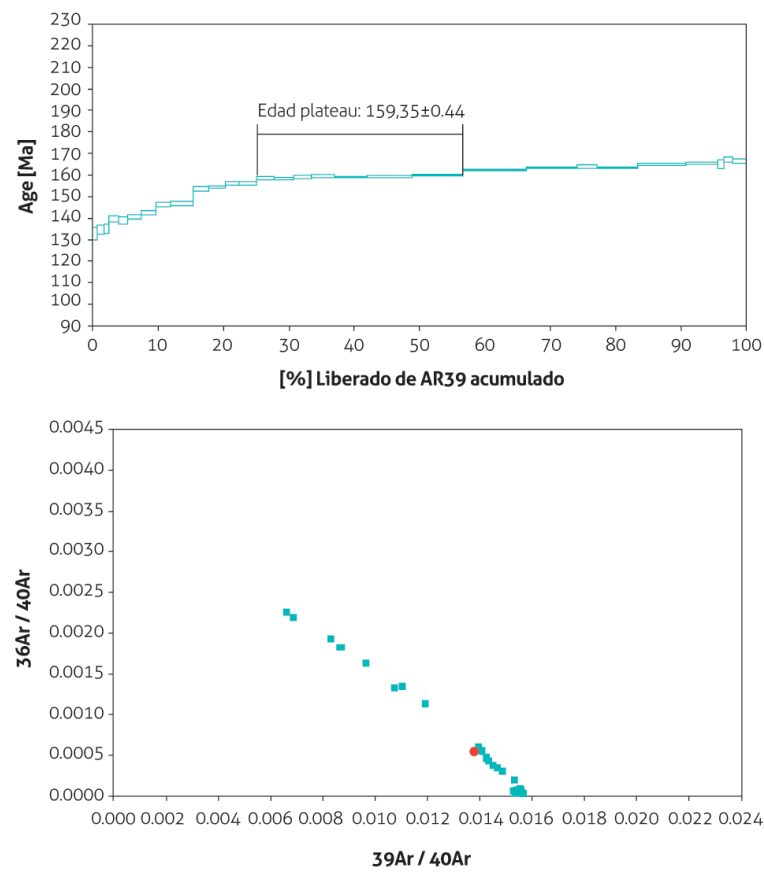

Figura 8. Espectro de edad e isócrona inversa para la muestra GR-6613, Fuente: autor reportado por el Laboratorio de la Universidad de Oregón

El $\mathrm{Na}_{2} \mathrm{O}$ y el $\mathrm{K}_{2} \mathrm{O}$, en las muestras de pórfido, es alto, mayor en términos generales que las lavas de la formación Saldaña para contenidos similares de $\mathrm{SiO}_{2}$ (figura 5); algunas rocas presentan fenocristales de feldespato alcalino y alteración de la plagioclasa a saussurita y arcilla y del olivino a serpentina, como se observa en las secciones delgadas. Presenta dispersión en los diagramas TAS y $\mathrm{SiO}_{2}$ vs $\mathrm{K}_{2} \mathrm{O}$ (figura 5), probablemente relacionada a la movilidad de $\mathrm{Na}$ y K por alteración, aunque los valores por pérdidas por ignición (LOI) son $<2 \%$. Los fenocristales de plagioclasa + olivino + clinopiroxeno +/- ortopiroxeno +/- feldespato alcalino son normales en rocas de series alcalinas, lo que apoya el resultado de algunas muestras que se grafican en el campo de las series alcalinas en el diagrama TAS, con valores de $\mathrm{Na}_{2} \mathrm{O}-2,0<\mathrm{K}_{2} \mathrm{O}$, de $\mathrm{Na}_{2} \mathrm{O}+\mathrm{K}_{2} \mathrm{O}>6,6 \%$ y relación $\mathrm{K}_{2} \mathrm{O} / \mathrm{Na}_{2} \mathrm{O}>1$ (en la mayoría de muestras analizadas), similar al de series shoshoníticas.

Los cuerpos de pórfido son metaluminosos, menos diferenciados que las lavas de la formación Saldaña que presentan mayores valores de $\mathrm{SiO}_{2}$, que en su mayoría se grafican en el campo peraluminoso, con menores valores de álcalis, Fe y Mg, y se clasifican en el campo de las dacitas y riolitas (figura 5).
En todas las muestras de pórfidos, el tren de los elementos traza y las tierras raras (REE) es subparalelo, lo cual sugiere un evento magmático común para los diferentes cuerpos, con anomalía negativa de $\mathrm{Nb}$, Ti y anomalía positiva de $\mathrm{Cs}, \mathrm{Rb}, \mathrm{Ba}, \mathrm{Th}, \mathrm{Sr}, \mathrm{K}, \mathrm{y} \mathrm{Pb}$; además, presenta relaciones $\mathrm{Eu} / \mathrm{Eu}^{*}>1$, que se atribuyen a que no se dio fraccionamiento de la plagioclasa, y la relación $(\mathrm{La} / \mathrm{Yb})_{\mathrm{N}}$ varía entre 7 y 12 , que sugiere aporte mantélico y cortical. No muestra grandes diferencias con el tren de REE de las lavas de la formación Saldaña, pero algunos contenidos marcan diferencias, como el $\mathrm{P}$ y Ti, que son mayores en las muestras de pórfido y afines con series alcalinas que tienen mayor contenido de estos elementos, lo que sugiere diferencias entre las fuentes de magmas de los pórfidos y de las lavas de la formación Saldaña, probablemente debido a una mayor inclinación de la placa que subduce.

Los cuerpos de pórfidos se encuentran enriquecidos en tierras raras livianas (LREE), con valores de lantano (La) mayores a cien veces el condrito de Nakamura (1974), patrón homogéneo que se va empobreciendo progresivamente hacia los elementos más pesados (HREE), y una marcada pendiente negativa, comportamiento que es normal en rocas generadas en ambiente de arco de margen continental.

Las relaciones estratigráficas de los cuerpos de pórfido descritos en este trabajo, con lavas y tobas de la formación Saldaña y plutones de la edad Jurásica Inferior, indican intrusión y emplazamiento posterior, que está en concordancia con la edad obtenida por el método Ar-Ar.

La edad obtenida por el método Ar-Ar en plagioclasa presenta una meseta ligeramente creciente, problemática para determinar el mejor plateau. Esta edad representa la edad de cierre de los fenocristales de plagioclasa entre 100 y $300{ }^{\circ} \mathrm{C}$, que ocurrió hace unos $160 \mathrm{Ma}$, con posterioridad a las edades de cristalización $\mathrm{U} / \mathrm{Pb}$ de los pulsos magmáticos descritos por Rodríguez et al. (2015a, 2017a, 2018), en lavas y tobas de la formación Saldaña y cuerpos plutónicos, que cristalizaron entre 195 y $168 \mathrm{Ma}$.

No hay correlación en edad y composición entre los cuerpos de pórfido descritos en este trabajo y las lavas y tobas de la formación Saldaña. Además, presentan una clara relación intrusiva, lo que permite concluir que estos cuerpos de pórfido no son parte de la formación Saldaña, pero podrían ser un pulso tardío del mismo arco que generó las lavas de la formación Saldaña y los plutones jurásicos del VSM. 


\section{Modelo evolutivo}

A continuación se propone un modelo evolutivo, que se esquematiza en la figura 9.

195 a 168 Ma. Comienza una distensión de la margen continental que provoca la formación de cuencas de retroarco (back-arc) semejante a la interpretación de Toussaint (1995). Ocurren de manera simultánea vulcanismo y plutonismo de arco, así como sedimentación. Se originan secuencias volcanosedimentarias tales como la formación Saldaña y las vulcanitas de Pitalito en el VSM. El plutonismo genera en orden cronológico aproximado los plutones occidentales en el VSM, plutones intermedios entre los occidentales y orientales y los plutones orientales del VSM.
168 Ma a $158 \mathrm{Ma}$. Choque de terrenos alóctonos entre $\sim 168$ y $\sim 154$ Ma contra la margen continental conformada por basamento neoproterozoico, con el consecuente metamorfismo que forma el conjunto metamórfico neises y anfibolitas de Tierradentro (Rodríguez et al., 2017d) $(\sim 167 \sim 154 \mathrm{Ma})$, parte SW del complejo Cajamarca ( 158 y $\sim 147$ Ma, según Blanco-Quintero et al., 2014) y el complejo La Cocha-río Téllez ( 163 Ma, Zapata et al., 2017). Este choque causó el estrangulamiento de la placa subducida, lo que aumentó la inclinación y el posterior colapso del arco; además, se formaron los últimos pulsos del arco que corresponden a pórfidos alcalinos, lo cual cambió la composición y la posición de los plutones más cerca de la trinchera.

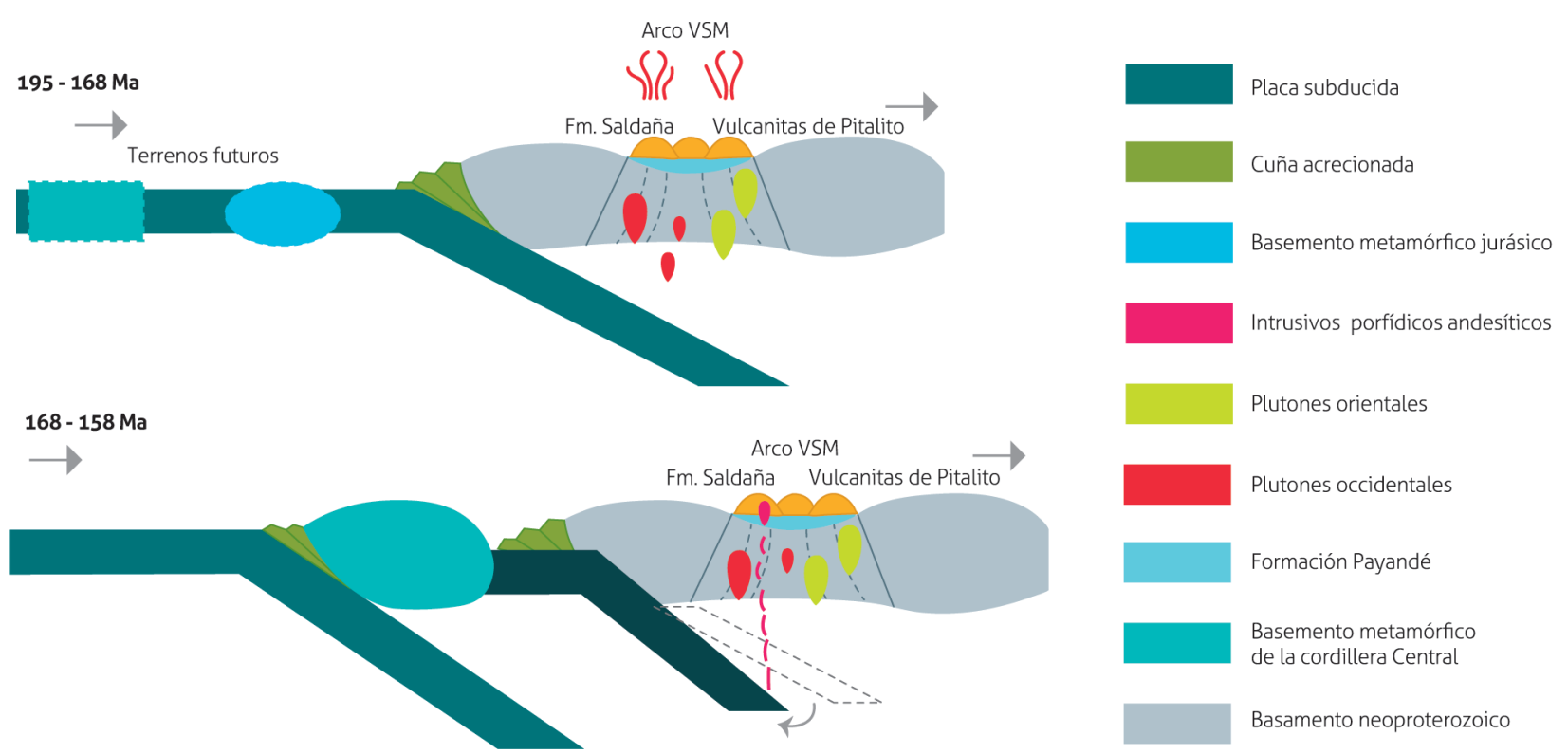

Figura 9. Modelo de evolución geológica para el VSM durante el Jurásico Fuente: autor

\section{Agradecimientos}

Se agradece al Servicio Geológico Colombiano, entidad que suministró los recursos para esta investigación, a los geólogos María Isabel Arango, José Gilberto Bermúdez y Juan Pablo Zapata, que colaboraron con el muestreo de rocas y en la elaboración de figuras; al geólogo Tomás Correa, quien realizó la traducción del resumen, y a los evaluadores que motivaron cambios en el manuscrito, que mejoraron el texto final.

\section{REFERENCIAS BIBLIOGRÁFICAS}

Álvarez, J. (1983). Geología de la cordillera Central y el occidente colombiano y petroquímica de los intrusivos granitoides meso-cenozoicos. Boletín Geológico, 26(2), 1-175.

Arango, M., Rodríguez, G., Bermúdez, J. y Zapata, G. (2015). Catálogo de unidades litoestratigráficas de Colombia: cuarzomonzonita de Anchique, cordillera Central, Huila-Tolima. Bogotá: Servicio Geológico Colombiano. 
Aspden, J., McCourt, W. y Brook, M. (1987). Geometrical control of subduction-related magmatism: the Mesozoic and Cenozoic plutonic history of Western Colombia. Journal of the Geological Society of London, 144(6), 893-905. Doi: 10.1144/gsjgs.144.6.0893.

Bermúdez, J., Arango, M., Rodríguez, G. y Zapata, G. (2015). Catálogo de unidades litoestratigráficas de Colombia: cuarzomonzodiorita de San Cayetano, cordillera Central, Tolima. Bogotá: Servicio Geológico Colombiano.

Blanco Quintero, I., García Casco, A., Toro, L., Moreno, M., Ruiz, E., Vinasco, C., et al. (2014). Late Jurassic terrane collision in the northwestern margin of Gondwana (Cajamarca complex, eastern flank of the Central Cordillera, Colombia). International Geology Review, 56(15), 1852-1872. Doi: 10.1080/00206814.2014.963710.

Bustamante, C., Cardona, A., Bayona, G., Mora, A., Valencia, V. y Gehrels, G. (2010). U-Pb LA-ICP-MS Geochronology and regional correlation of middle Jurassic intrusive rocks from the Garzon massif, upper Magdalena Valley and Central Cordillera, Southern Colombia. Boletín de Geología, 32(2), 93-109.

Carvajal, C., Fuquen, J. y Gómez, L. (1993). Geología de la plancha 282-Chaparral. Mapa. Bogotá: Ingeominas.

Carvajal, C., Fuquen, J, Gómez, L. y Núñez, A. (1983). Cartografía geológica y prospección geoquímica regional plancha 282-Chaparral. Memoria. Bogotá: Ingeominas.

Cediel, F., Mojica, J. y Macía, C. (1980). Definición estratigráfica del Triásico en Colombia, Suramérica. Formaciones Luisa, Payandé y Saldaña. Newsletters on Stratigraphy, 9(2), 73-104.

Cediel F., Mojica, J. y Macía, C. (1981). Las formaciones Luisa, Payandé y Saldaña: sus columnas estratigráficas características. Geología Norandina, (3): 11-19.

Cediel, F., Shaw, R. y Cáceres, C. (2003). Tectonic assembly of the Northern Andean block, in the circum-gulf of Mexico and the Caribbean: hydrocarbon habitats, basin formation and plate tectonics. AAPG Bulletin, (79), 815-848.

Cochrane, R., Spikings, R., Gerdes, A., Winkler, W., Ulianov, A. y Mora, A. (2014). Distinguishing between in-situ and accretionary growth of continents along active margins. Lithos, 202-203, 382-394. Doi: 10.1016/j.lithos.2014.05.031.
Cossio, U., Rodríguez, G. y Rodríguez, M. (1994). Mapa geológico de la plancha 283-Purificación, departamento Tolima. Escala 1:100.000. Con memoria explicativa. Bogotá: Ingeominas.

Ferreira, P., Núñez, A. y Rodríguez, M. (2002). Memoria explicativa levantamiento geológico de la plancha 323 Neiva. Escala 1:100.000. Bogotá: Ingeominas.

Fuquen, J. y Núñez, A. (1989). Memoria explicativa del mapa geológico generalizado del departamento del Huila: geología, recursos minerales y amenazas geológicas. Bogotá: Ingeominas.

Fuquen, J., Rodríguez, G. y Cossio, U. (1989). Geología de la plancha 302 Aipe. Bogotá: Ingeominas.

Gendall, I., Quevedo, L., Sillitoe, R., Spencer, R., Puente, C. y León, J. (2000). Discovery of a Jurassic porphyry copper belt, Pangui area, Southern Ecuador. SEG Newsletter, 43(1), 8-15.

Geyer, O. (1973). Das präkretazische Mesozoikum von Kolumbien. Journal of the Czech Geological Society, (5), 1-156.

Goldsmith, R., Marvin, R. y Mehnert, H. (1971). Radiometric ages in the Santander Massif, Eastern Cordillera, Colombian Andes. U. S. Geological Survey Professional Paper, 750, D44-D49.

Grosse, E. (1935). Acerca de la geología del sur de Colombia. Informe rendido al Ministerio de Industrias sobre un viaje al Huila y alto Caquetá. Cegoc, (3).

Jiménez Mejía, D., Juliani, C. y Cordani, U. (2006). P-T-t conditions of high-grade metamorphic rocks of the Garzon Massif, Andean basement, SE Colombia. Journal of South American Earth Science, 21(4), 322-336.

Ibáñez Mejía, M., Ruiz, J., Valencia, V., Cardona, A., Gehrels, G. y Mora, A. (2011). The Putumayo origen of Amazonia and its implications for Rodinia reconstructions: New U-Pb geochronological insights into the Proterozoic tectonic evolution of Northwestern South America. Precambrian Research, 191(1-2), 5877. Doi: 10.1016/j.precamres.2011.09.005.

Koppers, A. (2002). ArArCALC-Software for 40Ar/39Ar age calculations. Computers \& Geosciences, 28(5), 605-619. Doi: 10.1016/S0098-3004(01)00095-4.

Kuiper, K., Deino, A., Hilgen, F., Krijgsman, W., Renne, P. y Wijbrans, J. (2008). Synchronizing rock clocks of Earth history. Science, 320(5875), 500-504. Doi: 10.1126/science.1154339. 
Kroonenberg, S. y Diederix, H. (1982). Geology of South-Central Huila, Uppermost Magdalena Valley, Colombia: A preliminary note. En Guide Book 21 Annual Field Trip. Bogotá: Asociación Colombiana de Geólogos y Geofísicos del Petróleo.

Leal Mejía, H. (2011). Phanerozoic gold metallogeny in the Colombian Andes: A tectono- magmatic approach (tesis de doctorado). Universitat de Barcelona, Barcelona, España.

Le Bas, M., Le Maitre, R., Streckeisen, A. y Zanettin, B. (1986). A chemical classification of volcanic rock based on total silica diagram. Journal of Petrology, 27(3), 745-750. Doi: 10.1093/petrology/27.3.745.

Le Maitre, R. (Ed.), Streckeisen, A., Zanettin, B., Le Bas, M., Bonin, B. y Bateman, P. (2002). Igneous rocks: A classification and glossary of terms, recommendations of the International Union of Geological Sciences, Subcommission of the Systematics of Igneous Rocks. Cambridge: Cambridge University Press.

Mantilla, F., Bissig, T., Valencia, V. y Craig, H. (2013). The magmatic history of the Vetas-California mining district; Santander Massif, Eastern Cordillera, Colombia. Journal of South American Earth Sciences, 45, 235-249.

McCourt, W., Feininger, T. y Brook, M. (1984). New geological and geochronological data from the Colombian Andes: Continental growth by multiple accretion. Journal of the Geological Society, (141), 831-845. Doi: 10.1144/gsigs.141.5.0831.

Meschede, M. y Frisch, W. (1998). Tectonic model for the Mesozoic and Early Cenozoic history of the Caribbean Plate. Tectonophysics, 296(3-4), 269-291. Doi: 10.1016/S0040-1951(98)00157-7.

Min, K., Mundil, R., Renne, P. y Ludwig, K. (2000). A test for systematic errors in ${ }^{40} \mathrm{Ar} /{ }^{39} \mathrm{Ar}$ geochronology through comparison with $\mathrm{U} / \mathrm{Pb}$ analysis of a 1.1-Ga rhyolite. Geochimica et Cosmochimica Acta, 64(1), 7398. Doi: 10.1016/S0016-7037(99)00204-5.

Mojica, J., Kammer, A. y Ujueta, G. (1996). El Jurásico del sector noroccidental de Suramérica y guía de la excursión al valle superior del Magdalena (Nov. 1-4/95), regiones de Payandé y Prado, departamento del Tolima, Colombia. Geología Colombiana, 21, 3-41.

Mojica, J., Villarroel, C., Cuerda, A. y Alfaro, M. (1988). La fauna de graptolites de la formación El Hígado (Llanvirniano-Llandeiliano), serranía de Las Minas, valle superior del Magdalena, Colombia. Memorias $V$ Congreso Geológico Chileno, tomo II. Santiago de Chile.

Mojica, J. (1980). Observaciones acerca del estado actual del conocimiento de la formación Payandé (Triásico Superior), valle superior del río Magdalena, Colombia. Geología Colombiana, 11, 67-91.

Nakamura, N. (1974). Determination of REE, Ba, Fe, Mg, $\mathrm{Na}$ and $\mathrm{K}$ in carbonaceous and ordinary chondrites. Geochimica et Cosmochimica Acta, 38(5), 757-775. Doi: 10.1016/0016-7037(74)90149-5.

Pearce, J. (1996). User's guide to basalt Discrimination Diagrams. Short course notes. Association géologuique du Canada.

Pearce, J., Harris, N. y Tindle, A. (1984). Trace element discrimination diagrams for the tectonic interpretation of granitic rocks. Journal of Petrology, 25(4), 956983. Doi: 10.1093/petrology/25.4.956.

Peccerillo, A. y Taylor, T. (1976). Geochemistry of Eocene calc-alkaline volcanic rocks from Kastamonu area, Northern Turkey. Contributions to Mineralogy and Petrology, 58(1), 63-81. Doi: 10.1007/BF00384745.

Pindell, J. y Dewey, J. (1982). Permo-Triassic reconstruction of Western Pangaea and the evolution of the Gulf of Mexico-Caribbean region. Tectonics, 1(2): 179-211. Doi: 10.1029/TC001i002p00179.

Rodríguez, G., Arango, M., Zapata, G. y Bermúdez, J. (2017a). Caracterización magmática del Jurásico del valle superior del Magdalena y cuenca Putumayo. Bogotá: Servicio Geológico Colombiano.

Rodríguez, G., Arango, M., Zapata, G. y Bermúdez, J. (2018). Petrotectonic characteristics, geochemistry, and $\mathrm{U}-\mathrm{Pb}$ geochronology of Jurassic plutons in the Upper Magdalena Valley-Colombia: implications on the evolution of magmatic arcs in the NW Andes. Journal of South American Earth Sciences, 81, 10-30.

Rodríguez, G., Zapata, G., Arango, M. y Bermúdez, J. (2017c). Caracterización petrográfica, geoquímica y geocronología de rocas granitoides pérmicas al occidente de La Plata y Pacarní - Huila, valle superior del Magdalena-Colombia. Boletín de Geología, 39(1), 37-64.

Rodríguez, G., Obando, G., Correa Martínez, A., Zapata, G., Correa, T. y Obando, M. (2017d). Redefinición del bloque norte del batolito de Ibagué con base en 
nuevos datos de petrografía, litogeoquímica y geocronología U-Pb. Resumen XVI Congreso Colombiano de Geología y III Simposio de Exploradores. Santa Marta.

Rodríguez, G., Arango, M., Zapata, G. y Bermúdez, J. (2016). Formación Saldaña. Catálogo de unidades litoestratigráficas de Colombia. Bogotá: Servicio Geológico Colombiano.

Rodríguez, G., Arango, M., Zapata, G. y Bermúdez, J. (2015a). Características petrográficas, geoquímicas y edad U-Pb de los plutones jurásicos del valle superior del Magdalena. Póster presentado en el XV Congreso Colombiano de Geología.

Rodríguez, G., Arango M., Bermúdez, J. y Zapata, G. (2015b). Catálogo de unidades litoestratigráficas de Colombia: cuarzomonzonita de Los Naranjos-cordillera Central, Huila-Tolima. Bogotá: Servicio Geológico Colombiano.

Rodríguez, G., Zapata, G., Velásquez, M., Cossio, U. y Londoño, A. (2003). Geología de las planchas 367 Gigante, 368 San Vicente del Caguán, 389 Timaná, 390 Puerto Rico, 391 Lusitania (parte noroccidental) y 414 El Doncello. Memoria explicativa, escala 1:100.000. Bogotá: Ingeominas.

Rodríguez, G. y Fuquen, J. (1989). Geología y prospección geoquímica de la plancha 302-Aipe (Huila). Ibagué: Ingeominas.

Rodríguez, G. (1995a). Petrografía y microtexturas del grupo Garzón y el granito de anatexis de El Recreo, macizo de Garzón, cordillera Oriental-Colombia. Revista Ingeominas, (5), 17-36.

Rodríguez, G. (1995b). Petrografía del macizo de La Plata, departamento del Huila. Revista Ingeominas, (5), 5-16.

Shand, S. (1943). Eruptive rocks: Their genesis, composition, classification, and their relation to ore-deposits with a chapter on meteorite. New York: John Wiley y Sons.

Spikings, R., Cochrane, R., Villagómez, D., van der Lelij, R., Vallejo, C. y Winkler, W. (2015). The geological history of Northwestern South America: From Pangaea to the early collision of the Caribbean Large Igneous Province (290-75 Ma). Gondwana Research, 27(1), 95-139. Doi: 10.1016/j.gr.2014.06.004.

Steiger, R. y Jäger, E. (1977). Subcommission on geochronology: Convention on the use of decay constant in geo- and cosmochronology. Earth and Planetary Science Letters, 36(3), 359-362. Doi: 10.1016/0012821X(77)90060-7.

Stibane, F. y Forero, A. (1969). Los afloramientos del Paleozoico en La Jagua (Huila) y Río Nevado (Santander del Sur). Geología Colombiana, (6), 31-66.

Streckeisen, A. (1978). Classification and nomenclature of volcanic rocks, lamprophyres, carbonatites and melilitic rocks: recommendation and suggestions. Neues Jahrbuch für Mineralogie, Abhandlungen, 134, 1-14.

Sun, S. y Mcdonough, W. (1989). Chemical and isotopic systematics of oceanic basalts: implications for mantle composition and processes. En Sanders, A., y Norry, M. (Eds.). Magmatism in oceanic basins. Special Publication, vol. 42. London: Geological Society.

Taylor, J. (1997). An introduction to error analysis: The study of uncertainties in physical measurements. Mill Valley: University Science Book.

Toussaint, J. (1995). Evolución geológica de Colombia: Triásico-Jurásico. Medellín: Universidad Nacional de Colombia.

Trumpy, D. (1943). Pre-Cretaceous of Colombia. GSA Bulletin, 54(9), 1261-1304. Doi: 10.1130/GSAB-541281.

Van der Lelij, R. (2013). Reconstructing north-western Gondwana with implications for the evolution of the Iapetus and Rheic Oceans: A geochronological, thermochronological and geochemical study (tesis de doctorado). Université de Genève, Ginebra, Suiza.

Van der Lelij, R., Spikings, R. y Mora, A. (2016). Thermochronology and tectonics of the Mérida Andes and the Santander Massif, NW South America. Lithos, 248-251, 220-239. Doi: 10.1016/j.lithos.2016.01.006.

Velandia, F., Ferreira, P., Rodríguez, G. y Núñez, A. (1996). Memoria explicativa levantamiento geológico de la plancha 366 - Garzón. Bogotá: Ingeominas.

Velandia, F., Morales, C., Caicedo, J. y Núñez, A. (1999). Geología de la plancha 345 - Campoalegre. Escala 1:100.000. Bogotá: Ingeominas.

Velandia, F. (2001). Memoria del mapa geológico del departamento del Huila. Escala 1:300.000. Bogotá: Ingeominas.

Velandia, F., Nuñez, A. y Marquínez, G. (2001). Mapa geológico Departamento del Huila. Escala 1:300.000. Memoria explicativa. Bogotá: Ingeominas. 
Villagómez, D., Spikings, R., Magna, T., Kammer, A., Winkler, W. y Beltrán, A. (2011). Geochronology, geochemistry and tectonic evolution of the Western and Central cordilleras of Colombia. Lithos, 125(3-4), 875-896. Doi: 10.1016/j.lithos.2011.05.003.

Villagómez, D., Martens, U. y Pindell, J. (2015). Are Jurassic and some older blocks in the Northern Andes in-situ or far-travelled? Potential correlations and new geochronological data from Colombia and Ecuador. Ponencia presentada en el simposio Tectónica Jurásica en la Parte Noroccidental de Sur América y Bloques adyacentes. Disponible en https://www.researchgate.net/ publication/298069969_Are_Jurassic_and_some_older_blocks_in_the_northern_Andes_in-situ_or_ far-travelled_Potential_correlations_and_new_geochronological_data_from_Colombia_and_Ecuador.

Villarroel, C. y Mojica, J. (1988). El Paleozoico Superior (Carbonífero Pérmico) sedimentario de Colombia: Afloramientos conocidos y características generales. Geología Colombiana, 16, 81-87.

Ward, D., Goldsmith, R., Jimeno, A., Cruz, J., Restrepo, H. y Gómez, E. (1973). Cuadrángulo H-12 Bucaramanga. Planchas 109 Rionegro - 120 Bucaramanga. Cuadrángulo H-13 Pamplona. Planchas 110 Pamplo- na - 121 Cerrito. Escala 1:100.000. Memoria explicativa. Boletín Geológico, 21(1-3), 1-133.

Winter, J. (2001). An introduction to igneous and metamorphic petrology. Upper Saddle River: Prentice Hall.

Winchester, J. y Floyd, P. (1977). Geochemical discrimination of different magma series and their differentiation products using immobile elements. Chemical Geology, 20, 325-343. Doi: 0.1016/00092541(77)90057-2.

Wood, D. (1980). The application of a ThHfTa diagram to problems of tectonomagmatic classification and to establishing the nature of crustal contamination of basaltic lavas of the British Tertiary Volcanic Province. Earth and Planetary Science Letters, 50(1), 11-30. Doi: 10.1016/0012-821X(80)90116-8.

York, D. (1969). Least squares fitting of a straight line with correlated errors. Earth and Planetary Science Letters, 5, 320-324. Doi: 10.1016/S0012-821X(68)80059-7.

Zapata, G., Rodríguez, G. y Arango, M. (2017). Petrografía, geoquímica y geocronología de rocas metamórficas aflorantes en San Francisco, Putumayo, y la vía Palermo-San Luis asociadas a los complejos La Cocha-río Téllez y Aleluya. Boletín Ciencias de la Tierra, 41, 48-65. Doi: 10.15446/rbct.n41.58630. 